\section{Retraction of the Drosophila germ band requires cell-matrix interaction}

\author{
Frieder Schöck ${ }^{1}$ and Norbert Perrimon ${ }^{2}$ \\ Department of Genetics, Howard Hughes Medical Institute, \\ Harvard Medical School, Boston, Massachusetts 02115, USA
}

Integrins and laminins are important mediators of cellmatrix interactions in both vertebrates and invertebrates. Here, we show that germ-band retraction in the Drosophila embryo, during which the tail end of the embryo retracts to its final posterior position, allows the investigation of cell spreading and lamellipodia formation in real time in vivo. We demonstrate that $\alpha 1,2$ laminin and $\alpha$ PS3 $\beta P S$ integrin are required for the spreading of a small group of cells of the amnioserosa epithelium over the tail end of the germ band. We further implicate a role for this spreading in the process of germband retraction.

Received December 17, 2002; revised version accepted January 22, 2003.

Integrins and laminins have been shown to function in signaling, in stable adhesion of differentiated epithelia to the basement membrane, and in transient adhesion during cell spreading and migration (Brown et al. 2000; De Arcangelis and Georges-Labouesse 2000; Schöck and Perrimon 2002b). Laminins are extracellular matrix molecules consisting of a long $\alpha$ chain and shorter $\beta$ and $\gamma$ chains arranged in a cross-like structure (Martin et al. 1999). One of their most prominent interaction partners are the integrins, which form transmembrane heterodimers of $\alpha$ and $\beta$ subunits. In Drosophila there are two $\alpha$, one $\beta$, and one $\gamma$ laminin genes, and five $\alpha$ and two $\beta$ integrin genes (Brown et al. 2000; Hynes and Zhao 2000; Fig. 1A). Laminins and integrins have partially overlapping, but not identical phenotypes, because, even though they bind to each other, they also have various nonshared interaction partners (Brown et al. 2000; De Arcangelis and Georges-Labouesse 2000). In Drosophila, several morphogenetic processes require the presence of integrins, for example the formation of muscle attachments and wing-blade adhesion (Roote and Zusman 1995; Brown et al. 2000). Similar to integrin mutants, mutations in Drosophila laminin are associated with wing-blade adhesion and muscle-development defects (Henchcliffe et al. 1993; Yarnitzky and Volk 1995; Martin et al. 1999). Further, both $\alpha 1,2$ laminin and $\beta P S$ integrin mutants show a transient twisting of the germ band (Roote and Zusman 1995; Martin et al. 1999).

[Keywords: Cell spreading; Drosophila; germ-band retraction; integrin; laminin]

Corresponding authors.

${ }^{1}$ E-MAIL fschoeck@genetics.med.harvard.edu; FAX (617) 432-7688.

${ }^{2}$ E-MAIL perrimon@rascal.med.harvard.edu; FAX (617) $432-7688$.

Article and publication are at http://www.genesdev.org/cgi/doi/10.1101/ gad.1068403.
Another process that is affected by the absence of integrins, but which has not been studied up to now, is germ-band retraction (Leptin et al. 1989; Walsh and Brown 1998). Germ-band retraction occurs in mid-embryogenesis after germ-band extension and prior to dorsal closure. It takes $\sim 2 \mathrm{~h}$ and involves large-scale epithelial movements, by which the tail end of the germ band, or embryo proper, moves to its final posterior position (Martinez Arias 1993). At the end of this process, the amnioserosa, a squamous epithelium, has spread out and covers the yolk sac on the dorsal side of the embryo. We have shown previously that the amnioserosa and the germ band move as one coherent sheet during retraction. In addition, amnioserosa cells undergo a dramatic shortening along their dorsoventral axis during retraction (Schöck and Perrimon 2002a). Most interestingly, retraction is associated with lamellipodia formation and cell spreading of the posterior cell row of the amnioserosa, which causes the amnioserosa to slightly overlap the tail end of the germ band (Schöck and Perrimon 2002a; Fig. $4 \mathrm{E}$, below). The lamellipodia of the amnioserosa spread on the apical extracellular matrix of the germ band, which is deposited by the end of embryonic stage 10 (Tepass and Hartenstein 1994).

To explore the mechanisms underlying the observed spreading of the amnioserosa, we analyzed mutants exhibiting germ-band retraction defects. Here, we show that $\alpha 1,2$ laminin and $\alpha$ PS3 $\beta P S$ integrin are required to generate the overlap of the amnioserosa over the tail end of the germ band, and that they interact genetically during retraction. Live imaging of embryos completely null for $\beta$ PS integrin revealed that lamellipodia formation is disrupted and that there is no cell-matrix adhesion between the amnioserosa and the tail end of the germ band.

\section{Results and Discussion}

\section{Analysis of maternal-effect U-shaped mutations}

To determine whether germ-band retraction is a suitable system to study cell-matrix interactions and lamellipodia formation, we searched for genes involved in the cellular functions required for retraction. We screened a collection of zygotic lethal mutations for mutants associated with maternal-effect U-shaped embryonic phenotypes (Perrimon et al. 1996; N. Perrimon, unpubl.). Ushaped cuticle phenotypes are characterized by the dorsal location of abdominal denticle belts $6-8$ and are indicative of a failure of retraction (Frank and Rushlow 1996). One of the mutants we uncovered, $4 Y 18$, shows a U-shaped phenotype when both the zygotic and the maternal contributions are removed using the germ-line clone method (Chou and Perrimon 1992; Fig. 1B,C). Mapping experiments revealed that $4 Y 18$ fails to complement mutations at the wing blister $(w b)$ locus, which encodes $\alpha 1,2$ laminin, a polypeptide of the trimeric laminins (Martin et al. 1999). Sequencing of PCR-amplified 4 Y18 genomic DNA revealed a mutation causing a truncation after 1427 amino acids, which deletes the trimerization domain of $\mathrm{Wb}$ (Fig. 1D). The mutant $\mathrm{Wb}$ protein appears to be unstable, because it was not detected with an $\mathrm{N}$-terminal anti-Wb antibody in homozygous mutant embryos (data not shown), suggesting that $w b^{4 Y 18}$ is a null allele. 


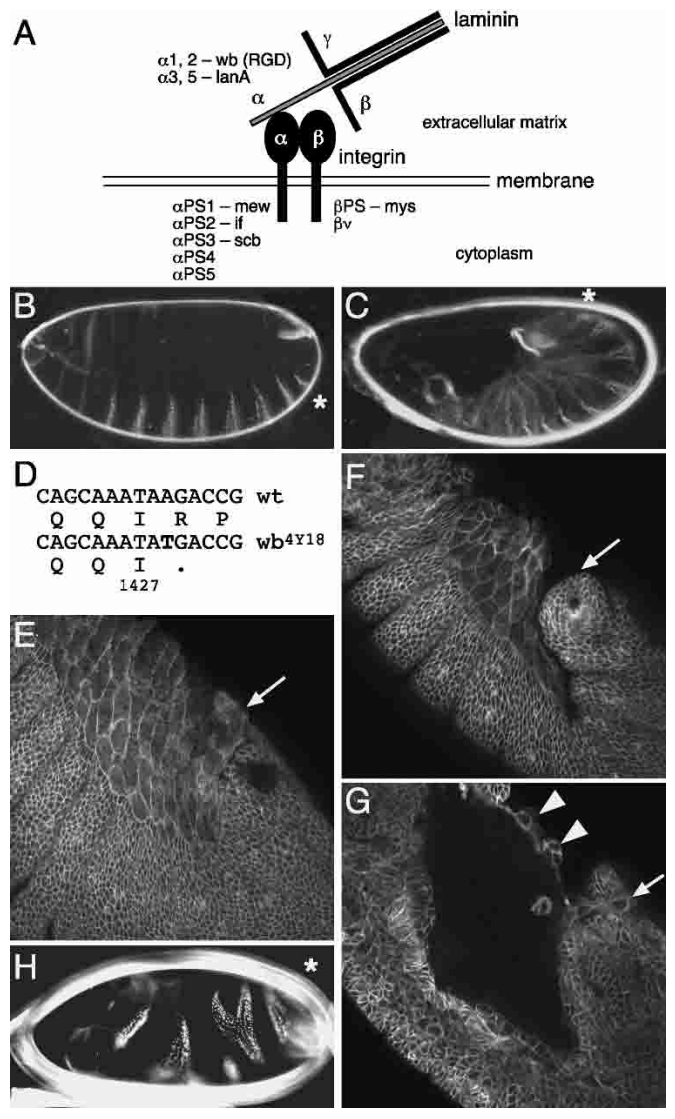

Figure 1. Mutation in $\alpha 1,2$ laminin disrupts germ-band retraction. (A) Mutations in a number of Drosophila integrin and laminin subunits are available as follows: if, inflated; $\operatorname{lan} A$, laminin $A_{;}$mew, multiple edematous wings; mys, myospheroid; scb, scab; wb, wing blister. Wb contains an arginine-glycine-aspartic acid (RGD) motif. The nomenclature of the Drosophila $\alpha$ laminin subunits is based on their similarities to the vertebrate $\alpha 1$ and $\alpha 2$ or $\alpha 3$ and $\alpha 5$ subunits, respectively (Martin et al. 1999). (B) Dark-field micrograph of the cuticle of a wild-type embryo with its distinctive ventral denticle belts. The position of abdominal segment eight (A8) in this panel, as well as in $C$ and $H$, is indicated by an asterisk. (C) A $w b^{4 Y 18}$ embryo that lacks both maternal and zygotic $(\mathrm{m} / \mathrm{z}) \alpha 1,2$ laminin activity does not undergo retraction. $(D)$ The $w b^{4 Y 18}$ mutation truncates the $\alpha 1,2$ laminin protein after amino acid position 1427. (E) Wild-type embryo at late stage 12 stained with anti- $\alpha$ Spectrin antibody. The amnioserosa slightly overlaps the tail end of the germ band (arrow; see also Fig. 4A). $(F, G)$ Anti- $\alpha$ Spectrin antibody staining of a late stage $12 w b^{4 Y 18} \mathrm{~m} / \mathrm{z}$ mutant embryo. A confocal surface section $(F)$ shows the lack of overlap of the amnioserosa over the tail end of the germ band (arrow). A confocal z-section 20- $\mu$ m deeper $(G)$ reveals multilayering of parts of the amnioserosa (arrowheads) and a few amnioserosa cells that still remain on top of the germ band (arrow). (H) lanA $A^{9-32} \mathrm{~m} / \mathrm{z}$ embryos have variable embryonic defects that range from poor cuticle differentiation to milder phenotypes, such as segment fusions/deletions and twisting (shown here).

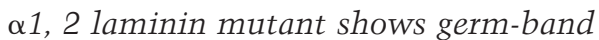 retraction defects}

Consistent with our finding that the $w b^{4 Y 18}$ maternal and zygotic $(\mathrm{m} / \mathrm{z})$ mutants exhibit U-shaped cuticle phenotypes, $\mathrm{m} / \mathrm{z}$ mutant embryos in the $\beta P S$ integrin myospheroid (mys) also show a U-shaped phenotype (Leptin et al. 1989; Fig. 5A, below). In addition, time-lapse recordings show that the U-shaped cuticle defect of $w b^{4 Y 18}$ and $\mathrm{mys}^{X G 43} \mathrm{~m} / \mathrm{z}$ mutant embryos results from a failure of germ-band retraction (data not shown; Fig. 3B, below).
At the cellular level, $w b^{4 Y 18} \mathrm{~m} / \mathrm{z}$ mutant embryos exhibit pleiotropic defects most evident in the amnioserosa. Anti- $\alpha$ Spectrin antibody stainings showed that, similar to mys $\mathrm{m} / \mathrm{z}$ mutants (see below), the overlap of the amnioserosa over the tail end of the germ band is defective, whereas cell-shape changes in the amnioserosa occur normally (Fig. 1, cf. F and wild type, E). In addition, the amnioserosa epithelium frequently exhibits multilayering, suggesting polarity defects (Fig. 1G, arrowheads). Laminin functions in epithelial polarity in other systems, for example during the development of the mammalian kidney epithelium, in which laminin is required to provide polarity cues (O'Brien et al. 2001). We cannot address where exactly $\alpha 1,2$ laminin is required to mediate germ-band retraction, because it is a secreted protein. However, the $w b^{4 Y 18}$ amnioserosa defects indicate that cell-matrix interactions are required between the amnioserosa and the underlying yolk sac and/or between the overlapping part of the amnioserosa and the apical extracellular matrix of the germ band.

We also analyzed the only other Drosophila $\alpha$ laminin, a3, 5 laminin, encoded by lamininA (IanA; Kusche-Gullberg et al. 1992; Henchcliffe et al. 1993; Martin et al. 1999). We generated $\mathrm{m} / \mathrm{z}$ mutant embryos of the null mutant lan $A^{9-32}$ to remove a weak maternal contribution that had not been analyzed previously (KuscheGullberg et al. 1992), and observed a range of defects. In more severe cases, embryos disintegrate before the onset of retraction, making it impossible to assess the contribution of $\operatorname{lan} A$ to germ-band retraction. However, in milder cases, lan $A \mathrm{~m} / \mathrm{z}$ embryos exhibit fusion or deletion of segments and twisting, but no retraction defects (Fig. 1H).

\section{$\alpha P S 3$ integrin mutant exhibits germ-band retraction defects and interacts with $\alpha 1,2$ laminin}

Another mutant (5/38) identified in our screen for maternal-effect U-shaped phenotypes, was mapped to the $s c a b(s c b)$ locus, which encodes the $\alpha$ PS3 integrin subunit (Stark et al. 1997; Fig. 2A). $\alpha$ PS3 integrin mutants have dorsal closure defects, and, in addition, $\alpha$ PS3 is expressed in morphogenetically active tissues, such as the salivary glands and the tracheal pits during their invaginations, as well as in the amnioserosa (Stark et al. 1997). To assess whether $\alpha$ PS3 zygotic expression, particularly in the amnioserosa, is required for retraction, we analyzed zygotic $s c b$ mutant embryos and found that they have U-shaped phenotypes as well. The penetrance of these phenotypes is low, but is enhanced (about threefold) in embryos transheterozygous for $s c b$ and a deficiency that deletes both $\alpha$ PS3 and the closely related aPS4 integrin (Fig. 2C), suggesting functional redundancy of these two closely related subunits. We have not yet assessed the contribution of the remaining two unrelated $\alpha$ PS integrin subunits, $\alpha$ PS1 and $\alpha$ PS2 .

To determine whether $\alpha 1,2$ laminin interacts with integrins in vivo, we tested for genetic interactions with a hypomorphic viable $\beta P S$ integrin allele $\left(m_{y s}{ }^{n j 42}\right)$ that shows a wing blister phenotype (Walsh and Brown 1998). Wing blisters arise due to a failure of cell-matrix interactions required for the apposition of two epithelia that form the wing blade (Walsh and Brown 1998). Consistent with the similarity of the mys and $w b$ embryonic phenotypes, removal of one copy of $w b$ in a hemizygous $\beta P S$ integrin mutant background results in a significant in- 




Figure 2. Mutation in $\alpha$ PS3 integrin disrupts germ-band retraction and interacts with $\alpha 1,2$ laminin. (A) Dark-field micrograph of the cuticle of a $s c b^{5738} \mathrm{~m} / \mathrm{z}$ embryo with defective germ-band retraction. A8 is indicated by an asterisk. (B) Homozygous mys ${ }^{\text {ni42 }}$ females were crossed to $w b^{4 Y 18} / \mathrm{CyO}$ or $l a n A^{9-32} / T M 3$ males. The number of hemizygous mys $^{\text {nj42 }}$ progeny with wing blisters is indicated by percent. (C) Frequency of germ-band retraction defects in various zygotic mutant backgrounds. Note the synergistic increase of retraction defects in $w b s c b$ double mutants compared with the respective single mutants, and the increase in the $s c b / s c b w b /+$ mutant. $(B, C)$ " $\mathrm{n}$ " refers to the number of flies or embryos scored. $(D)$ Quantification of spreading assay: $\alpha$ PS1, $\alpha$ PS $2 \mathrm{~m} 8$, or $\alpha$ PS 3 integrin genes were cotransfected with actin5CEGFP into nonadherent $\mathrm{S} 2$ cells and spread on indicated surfaces. The frequency of spread cells as a percentage of actin5CEGFP-transfected cells is shown. Bars indicate standard deviations from three independent experiments $>150$ cells counted in each experiment). (E-G) $\alpha$ PS integrin-transfected S2 cells plated on $\alpha 1,2$ laminin. Merger of green (cotransfection marker actin5CEGFP) and red (filamentous actin stained with AlexaFluor 568 phalloidin) channels. (E) S2 cells transfected with $\alpha$ PS1 integrin do not spread. S2 cells spread after transfection of $\alpha \operatorname{PS} 2 \mathrm{~m} 8(F)$ or aPS3 $(G)$ integrin.

crease in wing blisters (Fig. 2B), whereas removal of one copy of $\operatorname{lan} A$ does not cause an increase (Henchcliffe et al. 1993). We next used the low penetrance of germ-band retraction defects in $\alpha$ PS3 integrin mutants to test for genetic interactions with $\alpha 1,2$ laminin mutants during retraction. We analyzed zygotic $w b s c b$ double mutants and zygotic scb mutants with only one copy of $w b$. In both cases, we observed a significant increase in the frequency of U-shaped cuticle defects (Fig. 2C). These results show that $\alpha$ PS 3 integrin and $\alpha 1,2$ laminin interact in vivo during germ-band retraction.

\section{$\alpha P S 3 \beta P S$ integrin-containing S2 cells spread on $\alpha 1,2$ laminin}

We next asked whether $\alpha$ PS3 $\beta$ PS integrin is able to mediate cell-matrix interactions with $\alpha 1,2$ laminin, using a cell-spreading assay in tissue culture. Schneider's line 2
(S2) Drosophila cells are particularly suitable for such assays because, even though they express $\beta P S$ integrin, they do not spread due to the lack of expression of $\alpha \mathrm{PS}$ integrins (Gotwals et al. 1994). The $\beta P S$ integrin subunit heterodimerizes with $\alpha$ PS1, $\alpha$ PS2, and $\alpha$ PS3 integrin (Gotwals et al. 1994; Stark et al. 1997), and it was shown previously that $\alpha$ PS2 $\beta$ PS integrin interacts with $\alpha 1,2$ laminin in a cell-spreading assay (Graner et al. 1998). We therefore transfected S2 cells with $\alpha$ PS1, the splice variant $\alpha \mathrm{PS} 2 \mathrm{~m} 8$, or $\alpha \mathrm{PS} 3$ and plated them on plates coated with a $\mathrm{N}$-terminal $\alpha 1,2$ laminin fragment containing the RGD motif required for interaction with $\alpha$ PS $2 \beta P S$ to compare their adhesion behavior (Fig. 2D-G). Similar to $\alpha$ PS $2 \beta P S, \alpha$ PS3 $\beta$ PS mediates spreading on the N-terminal $\alpha 1,2$ laminin fragment, whereas $\alpha$ PS1 $1 \beta P S$ does not (Fig. 2D). To assess the contribution of the RGD motif in the observed spreading behavior, we tested spreading on a short fibronectin analog peptide containing the RGD motif (GRGDSPK). Only $\alpha$ PS2 $\beta$ PS exhibits spreading on this peptide, indicating that the contribution of the RGD motif to spreading of $\alpha$ PS3 $\beta$ PS is smaller than for $\alpha$ PS $2 \beta$ PS or is absent (Fig. 2D). Finally, all three integrin heterodimers may also bind to and spread on laminin domains not included in the $\alpha 1,2$ laminin fragment used for this spreading assay, as $\alpha$ PS1 $1 \beta P S$ integrin-containing cells had been shown previously to spread on full-length a3, 5 laminin (Gotwals et al. 1994). Altogether, our results demonstrate that $\alpha$ PS3 $\beta P S$ integrin mediates spreading on $\alpha 1,2$ laminin, that these integrin and laminin subunits are required for germ-band retraction, and that they interact genetically.

\section{$\beta P S$ integrin mutant retracts the germ band only partially}

To address more precisely the role of integrins during germ-band retraction, we used live imaging of $\beta P S$ integrin (mys) mutant embryos. We focused our analyses on mys mutants, because $\alpha$ integrins must heterodimerize with $\beta$ integrins in order to function, and mys $\mathrm{m} / \mathrm{z}$ mutants show the highest penetrance of U-shaped cuticle phenotypes. mys ${ }^{X 43} \mathrm{~m} / \mathrm{z}$ embryos are completely null for $\beta$ PS integrin (Leptin et al. 1989), and 65\% of the mutants display a severe U-shaped cuticle phenotype (Fig. $5 \mathrm{E}$, below). To outline all cells in time-lapse recordings, we marked embryos with ubiDE-cadherinGFP. In paternally rescued maternal mys mutant embryos, which were genotyped by the additional GFP expression in the amnioserosa, retraction proceeds normally as in wildtype embryos (Fig. 3A). In contrast, in mys $\mathrm{m} / \mathrm{z}$ mutant embryos, retraction progresses only for a short time, then stops prematurely, which results in an average of two to three abdominal segments remaining on the dorsal side (Fig. 3B). Most cell-shape changes are unaffected; in particular, amnioserosa cells still shorten along their dorsoventral axis (Fig. 3B; data not shown).

\section{$\beta P S$ integrin mutant does not adhere to the tail end of the germ band and does not form lamellipodia}

We then characterized the effects of $\beta P S$ integrin mutants on cell-matrix adhesion between the posterior part of the amnioserosa and the tail end of the germ band. In mys $\mathrm{m} / \mathrm{z}$ mutants, the amnioserosa no longer overlaps the germ band, but instead, crowds in front of the tail end of the germ band (Fig. 4, cf. B and A). At a slightly 


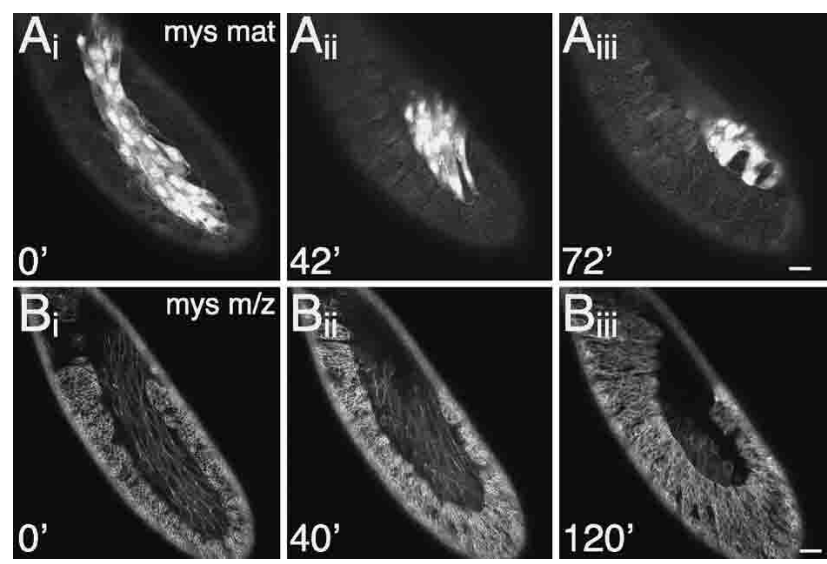

Figure 3. In the absence of $\beta$ PS integrin, the tail end of the germ band retracts only partially. $(A)$ Time-lapse recording of a lateral view of a paternally rescued mys ${ }^{X G 43}$ germ-line clone (GLC) embryo marked with FM7c, Kr-Gal4 UASGFP; ubiDE-cadherinGFP taken at 5 -min intervals. Retraction proceeds normally as in wild-type embryos. The additional GFP expression in the amnioserosa in $A$ compared with $B$ is due to $K r$-Gal4 UASGFP, which marks this embryo as paternally rescued. $(B)$ Time-lapse recording of a lateral view of a mys ${ }^{X G 43} \mathrm{~m} / z$ embryo marked with ubiDE-cadherinGFP taken at 5-min intervals. Retraction fails, but cell-shape changes, particularly in the amnioserosa, occur normally. Embryos were staged by the development of the dorsal ridge, a structure developing at late stage 11 dorsal of the maxillary and labial segment. Bars, $20 \mu \mathrm{m}$.

later stage, the amnioserosa collapses below the tail end of the germ band and cuts in under the germ band to the level of the hindgut (Fig. 4C). The collapse of the amnioserosa below the germ band may be a result of the dorsoventral shortening of the amnioserosa, which normally occurs during retraction (Schöck and Perrimon 2002a). This phenotype occurs with the same penetrance as the U-shaped cuticle phenotype. To determine what causes the amnioserosa to not overlap the tail end of the germ band, we analyzed time-lapse recordings of dorsal views of mys mutants marked with $\mathrm{Kr}$-Gal4, UASsrcEGFP. Paternally rescued maternal mys mutants show normal lamellipodia formation and adhesion of the amnioserosa to the tail end of the germ band (Fig. 4E). However, analysis of mys $\mathrm{m} / \mathrm{z}$ mutant embryos revealed that lamellipodia are generally absent from the posterior row of amnioserosa cells (Fig. 4F), and if present, are very small and transient (Fig. 4D). Thus, cell-matrix adhesion of the amnioserosa to the apical extracellular matrix of the germ band is disrupted. In conclusion, in both $w b^{4 Y 18}$ and $m y s^{X G 43} \mathrm{~m} / \mathrm{z}$ mutants, the amnioserosa does not overlap the tail end of the germ band as in wild-type embryos, indicating a requirement for cell-matrix adhesion between amnioserosa and germ band to maintain the overlap.

\section{Expression of $\beta P S$ integrin in the amnioserosa rescues retraction defects}

Finally, to determine whether $\beta$ PS integrin in the amnioserosa is sufficient to mediate germ-band retraction, we rescued the U-shaped phenotype of mys $\mathrm{m} / \mathrm{z}$ mutants by expressing $\beta P S$ integrin in the amnioserosa with the Gal4/UAS system (Brand and Perrimon 1993). Expression of UASmys with the $\mathrm{Kr}$-Gal4 line, which drives expression in the amnioserosa and six segments of the germ band (T3-A5), rescues the U-shaped cuticle phenotype of mys $\mathrm{m} / \mathrm{z}$ mutants substantially (Fig. 5B,E). Notably, expression of UASmys exclusively in the amnioserosa with the LP1-Gal4 driver rescues the retraction defects of mys $\mathrm{m} / \mathrm{z}$ mutants to the same degree (Fig. 5C,E).
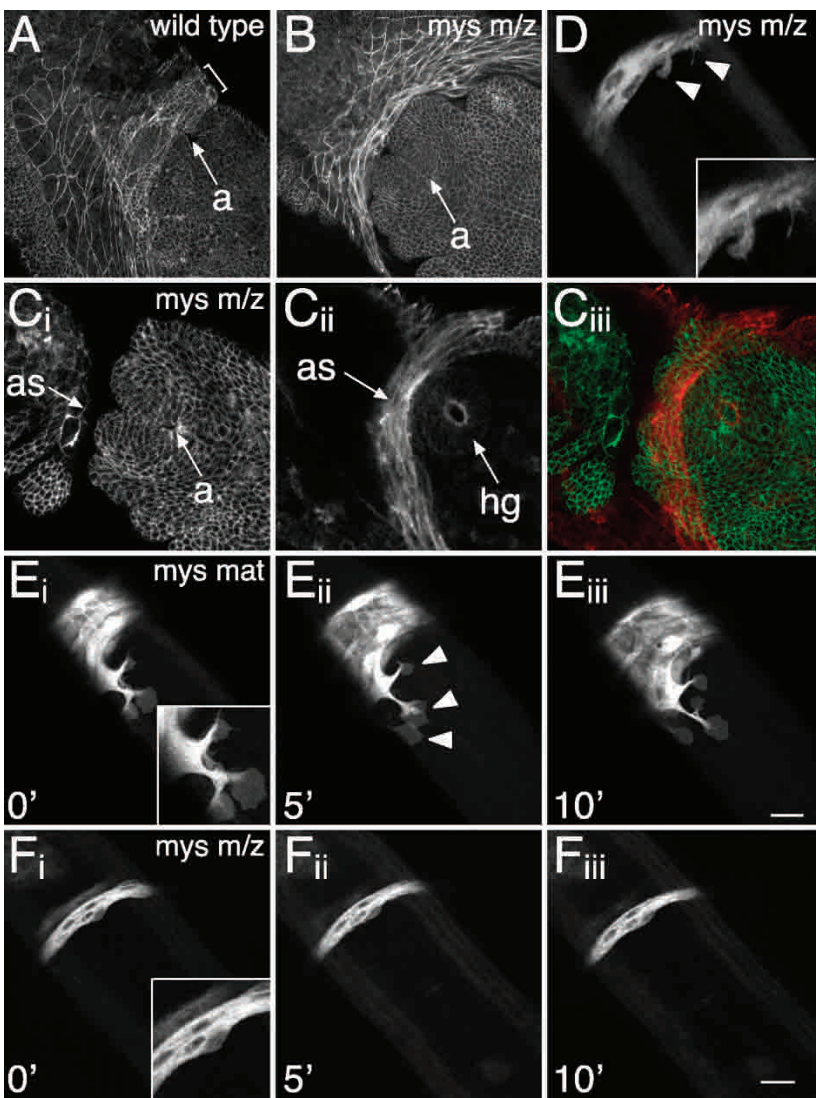

Figure 4. In the absence of $\beta$ PS integrin, the amnioserosa does not form lamellipodia and fails to adhere to the germ band. (A) Dorsal view of a wild-type embryo marked with ubiDE-cadherinGFP and fixed at early embryonic stage 12 to illustrate the overlap of the amnioserosa over the germ band. Bracket indicates the area of overlap. Overlay of three confocal z-sections. $(B, C)$ Dorsal view of mys ${ }^{X G 43} \mathrm{~m} / \mathrm{z}$ embryos marked with ubiDE-cadherinGFP and fixed at early and late embryonic stage 12 , respectively. $(B)$ The amnioserosa crowds in front of the tail end of the germ band; it does not overlap the anal pads (compare with $A$ ). (C) At a slightly later stage, the amnioserosa collapses below the germ band to the level of the hindgut. A surface z-section $\left(C_{i}\right)$ and an overlay of four z-sections 20- $\mu \mathrm{m}$ deeper $\left(C_{i i}\right)$ are shown. $\left(C_{i i i}\right)$ The merge, in which $C_{i i}$ was pseudocolored in red to illustrate the location of the amnioserosa below the tail end of the germ band. $(D)$ One frame of a time-lapse recording of a dorsal view of a mys $s^{X G 43} \mathrm{~m} / \mathrm{z}$ embryo marked with $\mathrm{Kr}$-Gal4 UASsrcEGFP as in F. Sometimes small protrusions are visible that look abnormal and collapse quickly. (E) Time-lapse recording of a dorsal view of a paternally rescued mys ${ }^{X G 43}$ GLC embryo marked with FM7c, Kr-Gal4 UASGFP; Kr-Gal4 UASsrcEGFP. The amnioserosa extends lamellipodia over the tail end of the germ band, and adheres to the germ band as in wild-type embryos. The additional nuclear GFP expression in the amnioserosa in $E$ compared with $D$ and $F$ marks this embryo as paternally rescued. Insets in $D, E$, and $F$ show enlarged views. $(F)$ Time-lapse recording of a dorsal view of a mys ${ }^{X G 43} \mathrm{~m} / \mathrm{z}$ embryo marked with $\mathrm{Kr}$-Gal4 UASsrcEGFP. Note the complete absence of lamellipodia. Amnioserosa spreading over the apical extracellular matrix of the germ band is disrupted. The time-lapse recordings shown in $E$ and $F$ are at the same magnification and developmental stage. Embryos were staged by the development of the anus. Arrowheads mark lamellipodia. a, anus; as, amnioserosa; hg, hindgut. Bars, $20 \mu \mathrm{m}$. 


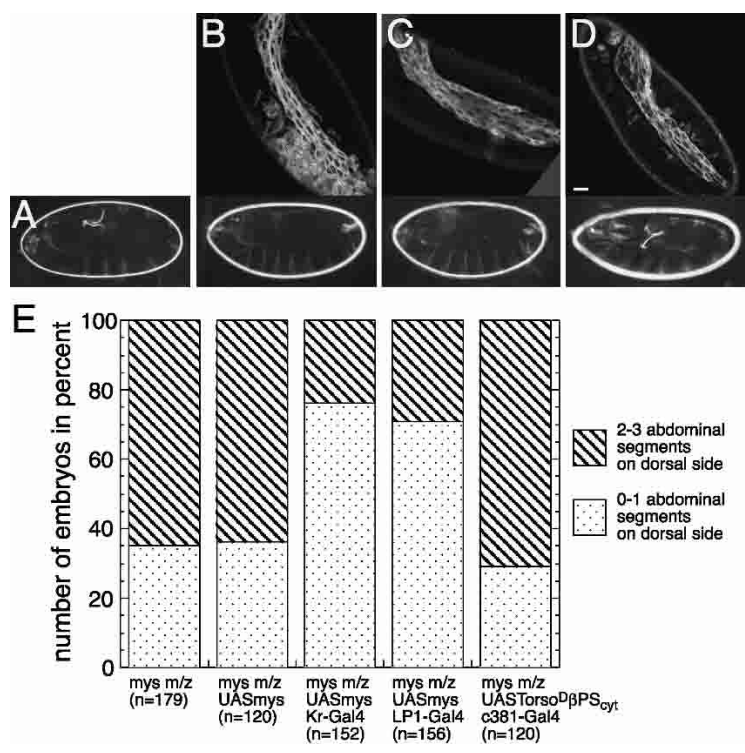

Figure 5. Expression of $\beta P S$ integrin in the amnioserosa rescues retraction defects. (A) mys $^{X G 43} \mathrm{~m} / \mathrm{z}$; UASmys. (B) mys ${ }^{X G 43} \mathrm{~m} / \mathrm{z}$; KrGal4 UASmys. (C) mys ${ }^{X G 43} \mathrm{~m} / \mathrm{z}$; LP1-Gal4 UASmys. (D) mys ${ }^{X G 43}$ $\mathrm{m} / \mathrm{z}$; c381-Gal4 UASTorso ${ }^{D} \beta P S_{c y t}$. The top row illustrates the expression of the Gal4 lines driving UASsrcEGFP. The bottom row depicts typical cuticles of embryos expressing the respective constructs. (E) Rescue efficiency: "n" refers to the number of embryos of the indicated genotype that were scored (see Materials and Methods). Bar, $20 \mu \mathrm{m}$.

In contrast, neither the UASmys construct alone, nor the amnioserosal expression of an adhesion-deficient $\beta P S$ integrin capable only of signaling (Martin-Bermudo and Brown 1999; Martin-Bermudo 2000) rescued the Ushaped phenotype (Fig. 5D,E). These data indicate that $\beta P S$ integrin expression is required in the amnioserosa, and that the adhesive properties of $\beta P S$ integrin are most likely required for retraction.

\section{Conclusions}

Altogether, we propose that in wild-type embryos, dorsoventral contraction of amnioserosa cells contributes to the extension of germ-band epithelial cells along the dorsoventral axis. This dorsoventral extension of the germband epithelium may then promote the retraction of the remaining segments that are still folded back. In the absence of proper laminin/integrin-mediated adhesion, the overlap of the amnioserosa over the tail end of the germ band is defective. We suggest that dorsoventral contraction of the amnioserosa in this mutant situation results in the collapse of the amnioserosa underneath the germ band, leading to an incorrect deployment of forces on the germ band and eventual complete or partial failure of retraction. We further note that the amnioserosa may also not adhere properly to the underlying yolk sac in the integrin mutants, which could also contribute to retraction defects. Our study demonstrates the involvement of $\alpha$ PS3 $\beta$ PS integrin and $\alpha 1,2$ laminin in germ-band retraction and provides a hypothesis for how retraction proceeds.

To date, studies of integrin function during lamellipodia formation and cell spreading have been mostly limited to tissue culture. A wealth of information has been obtained on the role of these molecules in the information flow from the extracellular matrix to the cytoskeleton during migration (Geiger et al. 2001). The process of germ-band retraction in the Drosophila embryo now provides an in vivo model system in which the function of the molecules identified in tissue culture experiments can be systematically tested. Uniquely, this system allows the study of lamellipodia formation and cell spreading in embryos mutant for candidate genes in real time and in vivo. Finally, the characterization of additional mutations associated with germ-band retraction defects will allow the identification of novel players involved in cell-matrix adhesion.

\section{Materials and methods}

\section{Fly strains}

The following fly strains were used: Kr-Gal4 40 , c381-Gal4, LP1-Gal4, UASmys, UASTorso ${ }^{D} \beta P S_{\text {cyt }}$ UASsrcEGFP, ubiDE-cadherinGFP, scb ${ }^{5 J 38}$ FRTG13, scb ${ }^{2}$, mys $^{X G 43}$ FRT101, mys $s^{n j 42}, \operatorname{lanA}^{9-32} F R T 79 D F$, and $w b^{4 Y 18}$ FRT40A (Leptin et al. 1989; Martin-Bermudo and Brown 1996, 1999; Oda and Tsukita 1999, 2000; Deng and Ruohola-Baker 2000; Herranz and Morata 2001). We made the following recombinants using standard genetic techniques: UASsrcEGFP $\mathrm{Kr}$-Gal4 40 , UASTorso $^{D} \beta P S_{c y t}$ UASmCD8GFP, UASmys UASsrcEGFP, and $w b^{4 Y 18} s c b^{2}$.

\section{Time-lapse recording}

Time-lapse recordings were done as described (Schöck and Perrimon 2002a). To analyze embryos, we used ubiDE-cadherinGFP, which is ubiquitously expressed and localizes to adherens junctions, thereby outlining cells (Oda and Tsukita 2001), and UASsrcEGFP, which localizes to both cytoplasm and plasma membrane due to the Src myristilation anchor attached to EGFP. mys germ-line clone (GLC) embryos were analyzed for lamellipodia by crossing FM7c, Kr-Gal4 UASGFP; UASsrcEGFP $\mathrm{Kr}$-Gal4 $40^{\circ}$ or FM7c, Kr-Gal4 UASGFP; ubiDE-cadherinGFP males to mys GLC females. This allowed us to distinguish paternally rescued embryos expressing nuclear GFP from $\mathrm{m} / \mathrm{z}$ embryos.

\section{Genetic studies}

From a GLC screen of more than 6000 zygotic lethal mutations (Perrimon et al. 1996; N. Perrimon, unpubl.), a number of mutants were isolated that exhibit U-shaped embryonic phenotypes. GLCs were produced in females of the genotype $y w$ hsFLP; mutant FRT/ovo ${ }^{D 1}$ FRT using the $O V O^{D 1}$ system and mated to mutant or wild-type males for determination of paternal rescue as described (Chou and Perrimon 1992).

$w b^{4 Y 18}$ and $s c b^{5 / 38}$ were mapped using deficiencies and alleles of the respective mutants. $4 Y 18$ was mapped to a small region $(35 \mathrm{~A})$ containing two loci that mutate to lethality; one of them, $w b$, did not complement 4Y18. As expected, the smallest deficiency $D f(2 L)$ el80f1 did not paternally rescue the $4 Y 18$ GLC mutant. The $w b^{4 Y 18}$ maternal effect U-shaped phenotype is partially penetrant and fully paternally rescueable. Twentytwo percent of the embryos from $w b^{4 Y 18}$ GLC females crossed to $w b^{4 Y 18} /+$ males show a U-shaped phenotype (A7 and A8 on dorsal side), whereas only $2 \%$ show this phenotype when $w b^{4 Y 18}$ GLC females were crossed to wild-type males; 5J38 was mapped similarly to 51D-52A, and failed to complement mutants at the $s c b$ locus.

For the rescue experiments, we crossed mys GLC females to $\mathrm{Kr}$-Gal4 UASsrcEGFP/CyO; UASmys/TM3, actGFP, Ser males or to UASmys UASsrcEGFP/+ LP1-Gal4/+ and UASTorso ${ }^{D} \beta P S_{c y t} U A S m C D 8 G F P /+$; c381-Ga14/+ males, respectively. We sorted aged embryos for the presence of Kr-Gal4 UASsrcEGFP and the absence of TM3, actGFP or for the presence of UASmys UASsrcEGFP and UASTorso ${ }^{D} \beta S_{\text {cyt }}$ $U A S m C D 8 G F P$, respectively. We then aged these embryos further to allow paternally rescued mys GLC embryos to hatch.

\section{Histochemistry and sequencing}

Primary antibody stainings with polyclonal rabbit anti-Wb 25632 (1:100) and monoclonal mouse anti- $\alpha$ Spectrin $(3 \Delta 9$ from DSHB, $1: 10)$ were performed as described (Pesacreta et al. 1989; Martin et al. 1999). Fluorescein-conjugated goat anti-rabbit IgG (Vector, 1:400) and AlexaFluor 488conjugated donkey anti-mouse IgG (Molecular Probes, 1:500) were used 
as secondary antibody. Genomic DNA of wild-type and $w b^{4 Y 18}$ homozygous mutant embryos was PCR-amplified with 16 primer pairs and sequenced by standard thermal cycle dideoxy sequencing.

\section{Cell-spreading assay}

The cell-spreading assay was performed as described (Zavortink et al. 1993). Briefly, a 340-amino-acid His-tagged RGD-containing fragment of $\alpha 1,2$ laminin was purified under denaturing conditions (Graner et al. 1998). A 3.6-kb XhoI SpeI full-length cDNA fragment of $\alpha$ PS3 was cloned into pMK33 (Gotwals et al. 1994; Stark et al. 1997). S2 cells were transiently transfected and expression of $\alpha$ PS integrins was induced with 500 $\mu \mathrm{M} \mathrm{CuSO}_{4}$ for $24 \mathrm{~h}$. Cells were allowed to spread for $6 \mathrm{~h}$ on coated 96 well tissue culture plates and then fixed for $30 \mathrm{~min}$ in $4 \%$ formaldehyde, and at the same time stained for filamentous actin with AlexaFluor 568 phalloidin (Molecular Probes, 1:50). Experiments were done in triplicate with at least 150 cells counted in each experiment.

\section{Acknowledgments}

We thank S. Baumgartner, D. Brower, N. Brown, T. Bunch, R. Hynes, G. Morata, H. Oda, H. Ruohola-Baker, E. Spana, and the Bloomington stock center for flies and reagents, and P. Bradley, R. DasGupta, and E. KüsterSchöck for comments on the manuscript. F.S. was an HHMI Fellow and is currently an HFSPO Long-Term Fellow. N.P. is an HHMI investigator.

The publication costs of this article were defrayed in part by payment of page charges. This article must therefore be hereby marked "advertisement" in accordance with 18 USC section 1734 solely to indicate this fact.

\section{References}

Brand, A. and Perrimon, N. 1993. Targeted gene expression as a means of altering cell fates and generating dominant phenotypes. Development 118: 401-415.

Brown, N.H., Gregory, S.L., and Martin-Bermudo, M.D. 2000. Integrins as mediators of morphogenesis in Drosophila. Dev. Biol. 223: 1-16.

Chou, T.B. and Perrimon, N. 1992. Use of a yeast site-specific recombinase to produce female germline chimeras in Drosophila. Genetics 131: 643-653.

De Arcangelis, A. and Georges-Labouesse, E. 2000. Integrin and ECM functions: Roles in vertebrate development. Trends Genet. 16: 389395.

Deng, W.M. and Ruohola-Baker, H. 2000. Laminin A is required for follicle cell-oocyte signaling that leads to establishment of the anteriorposterior axis in Drosophila. Curr. Biol. 10: 683-686.

Frank, L.H. and Rushlow, C. 1996. A group of genes required for maintenance of the amnioserosa tissue in Drosophila. Development 122: 1343-1352.

Geiger, B., Bershadsky, A., Pankov, R., and Yamada, K.M. 2001. Transmembrane crosstalk between the extracellular matrix and the cytoskeleton. Nat. Rev. Mol. Cell. Biol. 2: 793-805.

Gotwals, P.J., Fessler, L.I., Wehrli, M., and Hynes, R.O. 1994. Drosophila PS1 integrin is a laminin receptor and differs in ligand specificity from PS2. Proc. Nat1. Acad. Sci. 91: 11447-11451.

Graner, M.W., Bunch, T.A., Baumgartner, S., Kerschen, A., and Brower, D.L. 1998. Splice variants of the Drosophila PS2 integrins differentially interact with RGD-containing fragments of the extracellular proteins tiggrin, ten-m, and D-laminin 2. J. Biol. Chem. 273: 1823518241.

Henchcliffe, C., Garcia-Alonso, L., Tang, J., and Goodman, C.S. 1993. Genetic analysis of laminin A reveals diverse functions during morphogenesis in Drosophila. Development 118: 325-337.

Herranz, H. and Morata, G. 2001. The functions of pannier during Drosophila embryogenesis. Development 128: 4837-4846.

Hynes, R.O. and Zhao, Q. 2000. The evolution of cell adhesion. J. Cell Biol. 150: F89-F96.

Kusche-Gullberg, M., Garrison, K., MacKrell, A.J., Fessler, L.I., and Fessler, J.H. 1992. Laminin A chain: Expression during Drosophila development and genomic sequence. EMBO J. 11: 4519-4527.

Leptin, M., Bogaert, T., Lehmann, R., and Wilcox, M. 1989. The function of PS integrins during Drosophila embryogenesis. Cell 56: 401-408.

Martin, D., Zusman, S., Li, X., Williams, E.L., Khare, N., DaRocha, S.,
Chiquet-Ehrismann, R., and Baumgartner, S. 1999. wing blister, a new Drosophila laminin $\alpha$ chain required for cell adhesion and migration during embryonic and imaginal development. J. Cell Biol. 145: 191-201.

Martin-Bermudo, M.D. 2000. Integrins modulate the Egfr signaling pathway to regulate tendon cell differentiation in the Drosophila embryo. Development 127: 2607-2615.

Martin-Bermudo, M.D. and Brown, N.H. 1996. Intracellular signals direct integrin localization to sites of function in embryonic muscles. J. Cell Biol. 134: 217-226.

- 1999. Uncoupling integrin adhesion and signaling: The $\beta P S$ cytoplasmic domain is sufficient to regulate gene expression in the Drosophila embryo. Genes \& Dev. 13: 729-739.

Martinez Arias, A. 1993. Development and patterning of the larval epidermis of Drosophila. In The development of Drosophila melanogaster. (ed. M. Bate and A. Martinez Arias), pp. 517-608. Cold Spring Harbor Laboratory Press, Cold Spring Harbor, NY.

O'Brien, L.E., Jou, T.S., Pollack, A.L., Zhang, Q., Hansen, S.H., Yurchenco, P., and Mostov, K.E. 2001. Rac1 orientates epithelial apical polarity through effects on basolateral laminin assembly. Nat. Cell Biol. 3: 831-838.

Oda, H. and Tsukita, S. 1999. Dynamic features of adherens junctions during Drosophila embryonic epithelial morphogenesis revealed by a Dacatenin-GFP fusion protein. Dev. Genes Evol. 209: 218-225.

. 2001. Real-time imaging of cell-cell adherens junctions reveals that Drosophila mesoderm invagination begins with two phases of apical constriction of cells. J. Cell Sci. 114: 493-501.

Perrimon, N., Lanjuin, A., Arnold, C., and Noll, E. 1996. Zygotic lethal mutations with maternal effect phenotypes in Drosophila melanogaster. II. Loci on the second and third chromosomes identified by P-element-induced mutations. Genetics 144: 1681-1692.

Pesacreta, T.C., Byers, T.J., Dubreuil, R., Kiehart, D.P., and Branton, D. 1989. Drosophila spectrin: The membrane skeleton during embryogenesis. J. Cell Biol. 108: 1697-1709.

Roote, C.E. and Zusman, S. 1995. Functions for PS integrins in tissue adhesion, migration, and shape changes during early embryonic development in Drosophila. Dev. Biol. 169: 322-336.

Schöck, F. and Perrimon, N. 2002a. Cellular processes associated with germ band retraction in Drosophila. Dev. Biol. 248: 29-39.

- 2002b. Molecular mechanisms of epithelial morphogenesis. Annu. Rev. Cell Dev. Biol. 18: 463-493.

Stark, K.A., Yee, G.H., Roote, C.E., Williams, E.L., Zusman, S., and Hynes, R.O. 1997. A novel $\alpha$ integrin subunit associates with $\beta P S$ and functions in tissue morphogenesis and movement during Drosophila development. Development 124: 4583-4594.

Tepass, U. and Hartenstein, V. 1994. The development of cellular junctions in the Drosophila embryo. Dev. Biol. 161: 563-596.

Walsh, E.P. and Brown, N.H. 1998. A screen to identify Drosophila genes required for integrin-mediated adhesion. Genetics 150: 791-805.

Yarnitzky, T. and Volk, T. 1995. Laminin is required for heart, somatic muscles, and gut development in the Drosophila embryo. Dev. Biol. 169: 609-618.

Zavortink, M., Bunch, T.A., and Brower, D.L. 1993. Functional properties of alternatively spliced forms of the Drosophila PS2 integrin $\alpha$ subunit. Cell Adhes. Commun. 1: 251-264. 




\section{Retraction of the Drosophila germ band requires cell -matrix interaction}

Frieder Schöck and Norbert Perrimon

Genes Dev. 2003, 17:

Access the most recent version at doi:10.1101/gad.1068403

References This article cites 30 articles, 16 of which can be accessed free at: http://genesdev.cshlp.org/content/17/5/597.full.html\#ref-list-1

License

Email Alerting Receive free email alerts when new articles cite this article - sign up in the box at the top Service right corner of the article or click here.

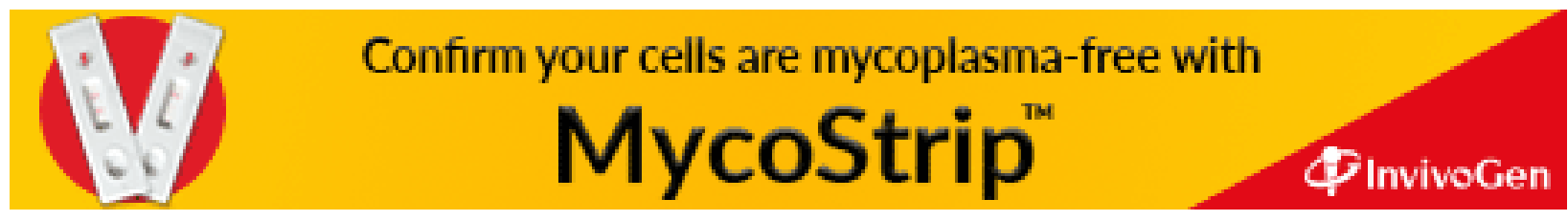

